\title{
Erosion Processes due to energetic particle-surface interaction
}

\author{
K. Schmid, J. Roth \\ Max-Planck-Institut für Plasmaphysik, EURATOM Association, BoltzmannstraSSe 2, 85748 \\ Garching b. München, Germany
}

\begin{abstract}
The interaction of the fast particles from the hot plasma of a magnetic confinement fusion experiment with the first wall is one of the most challenging problems toward the realization of a fusion power plant. The erosion of the first wall by the fast particles leads to life time limitations and the radiative cooling of the plasma by the eroded impurity species lowers the energy confinement. Apart from these obvious consequences also the trapping of large quantities of the fueling species (Deuterium and tritium) in re-deposited layers of the eroded species poses a problem due to accumulation of large radiative inventories and plasma fueling inefficiency. The source of all these challenges is the erosion of first wall components due to physical sputtering, chemical erosion and radiation enhanced sublimation. This paper will give an overview about the physical principles behind these erosion channels.
\end{abstract}

Keywords: Sputtering, Erosion

PACS: 61.80.Jh, 52.77.Dq, 52.70.Nc, 52.40.Hf

\section{INTRODUCTION}

In modern magnetic confinement fusion experiment experiments the modification of the first plasma exposed wall through the interaction with the fast particles, neutrals and ions is of key importance. Erosion of the first wall of course limits the life time of the first wall components but also leads to an influx of impurity species into the plasma. In the plasma the impurities emit line radiation and bremsstrahlung thereby cooling the plasma. While light impurities are fully ionized in the central plasma and only radiate bremsstrahlung heavy elements still have bound electrons that emit line radiation which leads to strong plasma cooling. The emitted Line radiation power scales with core charge $Z^{6}$ and therefore the maximum allowable impurity concentration $C_{M a x}$ for a burning fusion plasma is different for the various elements. For high- $\mathrm{Z}$ impurities like tungsten (W) $C_{\text {Max }} \approx 10^{-4} \%$ whereas for carbon $C_{\text {Max }} \approx 1 \%$.

Since the particle spectra varies across the poloidal circumference of the tokamak different materials are used depending on the particle fluxes and energies at a given poloidal location. Material is eroded at basically all plasma wetted areas and the eroded particles are transported through out the tokamak vacuum vessel and are finally re-deposited at a location usually far away of its initial point of erosion. Combined with the use of different elements leads to formation of mixed material layers at locations where the eroded particles are preferentially re-deposited. This mixed layer usually have properties that are very different from those of the pure material. In particular these re-deposited layers can be co-deposited with large amounts of hydrogen isotopes. This is particularly the case for the co-deposition of $\mathrm{C}$ with $\mathrm{H}$-isotopes where the layers can have $\mathrm{D} / \mathrm{C}$ ratios of 
up to $\approx 0.7$. This large trapped fuel inventory poses a severe radiation hazard due to the contained tritium in these layers.

The trigger for all of the above processes is the erosion of particle from the first wall due to interaction with the energetic particles from the plasma. This Erosion occurs by different processes: Physical sputtering which is the kinetic ejection of particles due to impact of energetic atoms or ions; Chemical erosion which is the formation of volatile compounds during exposure of a surface to a flux of reactive species; Chemical sputtering which is the formation of volatile compounds due to interaction of the reactive species with radiation damage produced by energetic particle bombardment; Radiation enhanced sublimation which the enhancement of sublimation by formation of weakly bonded defects at the surface during energetic particle bombardment at elevated temperatures.

The individual erosion processes have been studied extensively however a basic understanding can hardly be derived in fusion experiments themselves due to the large uncertainties in the particle fluxes, energies and surface temperatures. Therefore usually the erosion processes are investigated in more controlled laboratory experiments where the particle spectra in a fusion experiment are simulated by ion beams.

This paper will give an overview of physical basis of these four erosion processes.

\section{INTERACTION OF ENERGETIC PARTICLES WITH MATTER}

All erosion processes are part of the more general topic of interaction of energetic particles with matter. When an energetic projectile (neutral or charged) hits a solid its subsequent trajectory is defined by elastic scattering at the atoms inside the solid. This interaction is called "Nuclear Energy Loss". The projectile does not necessarily have to penetrate into the surface but can also be backscattered (三Reflection). When the projectile does penetrate the target the inelastic interaction with the target electrons leads to a continuous energy loss ("Electronic Energy Loss"). Both loss channels lead to dissipation of the projectiles initial energy. Once it has lost all its energy it gets stuck in the solid (三 Implantation).

The nuclear energy loss leads to formation of energetic recoil atoms in the solid which in turn can produce further recoils due to collision processes. From this collision cascade atoms can be emitted at the surface when their energy is large enough to overcome the surface binding energy ( $\equiv$ Physical sputtering). After the cascade has died down the solid my be left with permanent radiation damage (vacancies, interstitials etc.)

\subsection{Scattering}

The scattering processes in a collision cascade are characterized by two parameters: The energy transfer during the collision and the cross section for a particular scattering process. A particular elastic scattering process is defined by deflection angle $\theta$ of the projectile which is the angle between its initial and final velocity vector. The energy

transferred in a scattering event depends on $\theta$ and the mass ratio of projectile $M_{1}$ and 


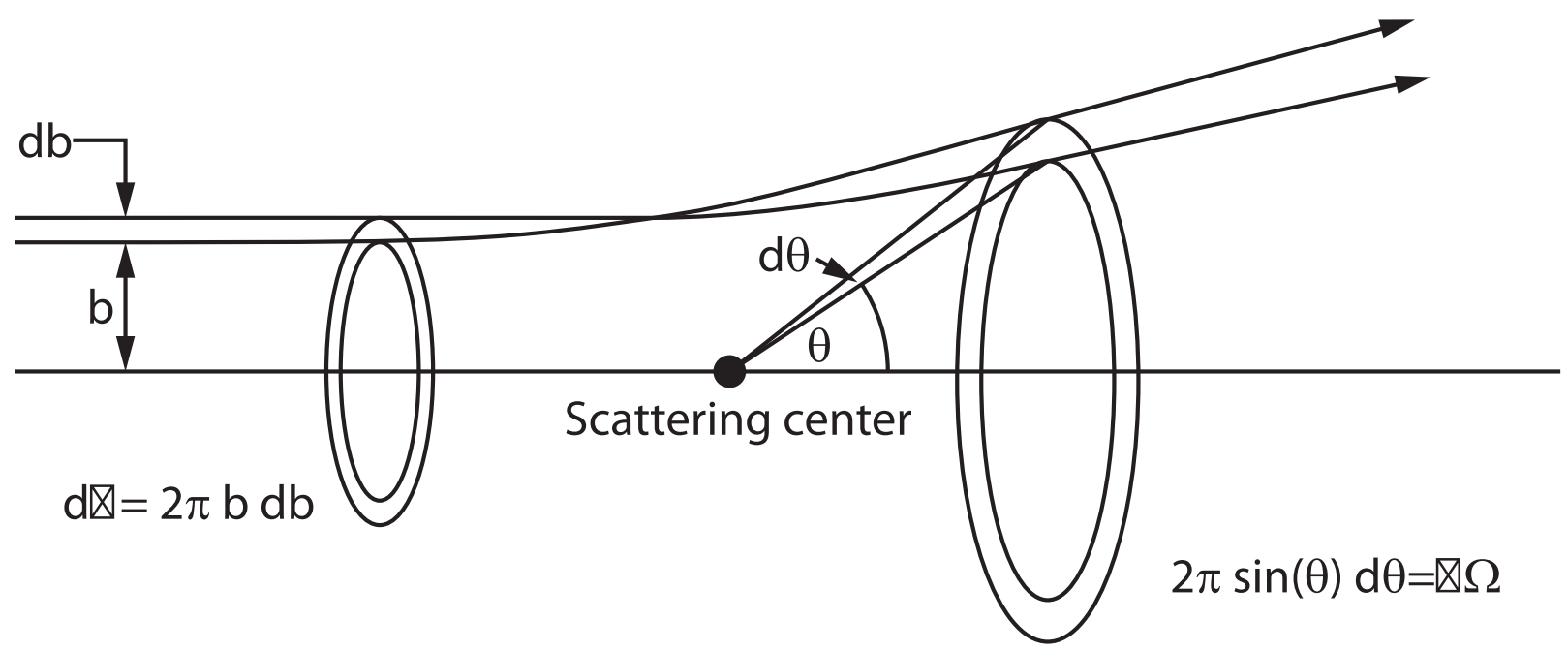

FIGURE 1. Geometrical definition of the differential scattering cross section $d \sigma / d \Omega$ for deflection by an angle $\theta$

target atom $M_{2}$.

$$
\frac{E_{1}}{E_{0}}=\left[\frac{\sqrt{M_{2}^{2}-M_{1}^{2} * \sin ^{2} \theta} \pm M_{1} * \cos \theta}{M_{2}+M_{1}}\right]^{2}
$$

bzw.

$$
\frac{E_{2}}{E_{0}}=\frac{4 M_{1} M_{2}}{\left(M_{1}+M_{2}\right)^{2}} * \cos ^{2} \phi
$$

In eq. 1 Where $E_{0}, E_{1}$ is the energy of the projectile before and after the scattering event. In eq. $2 E_{2}$ is the energy of target (recoil) atom after the scattering event. The angle $\phi$ in eq. 2 is the angle between the velocity vector of the projectile before and the recoil atom after the scattering event.

The scattering cross section is a measure of the probability for scattering a projectile by a given angle $\theta$ and a given areal density $m^{-2}$ of scattering centers. The differential scattering cross section $d \sigma / d \Omega$ denotes the area around the scattering center that has to be hit by the projectile in order to be deflected by and angle $\theta$ as visualized in Fig. 1 The differential scattering cross section can be calculated by assuming a certain scattering potential between the projectile and the target atom. Usually screened coulomb potentials are used where the screening term describes the shielding of the coulomb potential between the nuclei by the electrons. For very high energies the screening becomes negligible and an analytical expression for $d \sigma / d \Omega$ for a pure coulomb potential can be given [1].

$$
\frac{d \sigma}{d \Omega}=5.210^{-25}\left(\frac{Z_{1} Z_{2}}{E[k e V]}\right)^{2} \frac{\left[\sqrt{M_{2}^{2}-M_{1}^{2} \sin ^{2} \theta}+M_{2} \cos \theta\right]^{2}}{M_{2} \sin ^{4} \theta \sqrt{M_{2}^{2}-M_{1}^{2} \sin ^{2} \theta}}\left[10^{-25} \mathrm{~m}^{2}\right]
$$




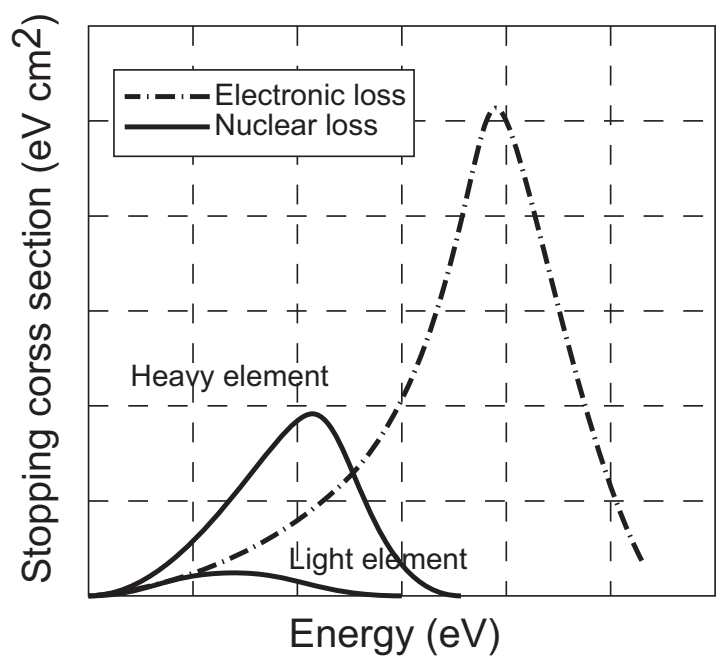

FIGURE 2. Nuclear and electronic stopping cross section as function of energy.

The scattering cross section scales with $Z^{2}$ and $E^{-2}$ so heavy elements at low energies are more likely to undergo large angle scattering than light and fast elements. This is also the reason why heavy projectiles produce large and dense collision cascades while light projectiles only undergo few collisions and do not produce dens cascades.

\subsection{Energy loss}

The energy loss of fast particles in matter is described by the stopping cross section $\varepsilon$ in units of $\left(\mathrm{eVm}^{2}\right)$. To obtain the loss of kinetic energy per distance traveled $\varepsilon$ has to be multiplied by the target atomic density $\left(\mathrm{m}^{-3}\right)$ to obtain the so called stopping power $\mathrm{S}(\mathrm{eV} / \mathrm{m}) . \varepsilon$ is a combination of nuclear and electronic stopping. The nuclear stopping cross section can be calculated by averaging the energy transfer for all $\theta$ weighed with the respective scattering cross section. The resulting expression scales with $\mathrm{Z}$ and $\mathrm{E}$ similar as the cross section in eq. 3 i.e for high energies due to the $E^{-2}$ dependence it becomes negligible.

The electronic energy loss is more complex since it includes the quantum mechanical interaction between the projectile and electrons in the solid. However two regimes can be distinguished: For low energies $(\mathrm{E}<25 \mathrm{keV} / \mathrm{amu})$ the electronic stopping cross section increases linearly with the projectile energy [2]. At high energies $\left(\mathrm{E}>1 / 2 M_{1} v_{F}^{2}\right.$, $v_{F}=$ Fermivelocity) the electronic stopping cross section decreases with $v^{-2}$ [3]. The general shape of the different contributions to $\varepsilon$ is shown in Fig. 1 As shown in Fig. 1 nuclear stopping only dominates at low energies and heavy (high-Z) projectiles at high energies electronic stopping dominates. This is also the reason why erosion by particle bombardment dominantly occurs at low energies (keV/amu) since there most of the projectile energy is transferred to nuclear energy loss i.e into the production of recoils which can leave the target as physically sputtered atoms. 


\section{PHYSICAL SPUTTERING}

Physical sputtering the is erosion of a target surface due to kinetic ejection of surface atoms during a collision cascade triggered by the impact of fast particle. Surface atoms can only leave the surface if their kinetic energy is high enough to overcome the surface binding energy (SBE). The SBE is usually taken to be identical to the heat of sublimation and is in the order of several $\mathrm{eV}$. In a fusion experiment the energy of particle varies from $\mathrm{eV}$ to $\mathrm{keV}$. Ions from the plasma have an energy determined by the ion temperature $\left(T_{i}\right)$ in the plasma and are further accelerated in the plasma boundary sheath potential (Debye Sheath) before they hit the first wall. Therefore the average impact energies of ions is calculated as in eq. 4 [4].

$$
\begin{aligned}
\left\langle E_{\text {ion }}\right\rangle & =3 T_{e} q+3 T_{i} \\
q & =\text { Ion charge state } \\
3 T_{e} & =\text { Debye Sheath potential for } \mathrm{H} \text { plasmas }
\end{aligned}
$$

In addition to the fast ions also energetic neutrals from charge exchange reactions hit the first wall. Therefore the energy spectra encountered in a magnetic fusion experiments is around $\mathrm{keV} / \mathrm{amu}$ which is just the range where the nuclear energy loss and thus also physical sputtering are most pronounced.

In Fig 3 the energy dependence of the physical sputter yield $Y_{P h y s}$ is shown for different projectile/target combinations. $Y_{P h y s}$ is defined as the number of eroded target atoms per impinging projectile. As described before physical sputtering is due to energy transfer to the target atoms i.e due to nuclear energy loss. Therefore one would expect the physical sputter yield to scale like the nuclear energy loss. While the general shape of all graphs in Fig 3 exhibit a nuclear energy loss like scaling, they all show the presence of threshold energy $E_{T h}$. This indicates that there is more to sputtering than just nuclear energy loss: For projectile energies below $E_{T h}$ the target atoms in the cascade can not get enough energies to overcome the surface binding energy and therefore no physical sputtering occurs. Therefore $E_{T h}$ depends on the mass ratio of projectile and target and on the SBE of the target. For light projectiles $E_{T h}$ depends strongly on the core charge $\mathrm{Z} 2$ of the target atoms and $Y_{P h y s}$ is similar for most target materials in the high energy range where the projectile energy is much larger than $E_{T h}$. This is due to the fact that for light ions most sputter events occur when the projectile is reflected in the surface and on its way out of the surface hits a target atom with a large enough energy transfer so that the target atom can overcome the SBE. Since reflection reflection involves a large angle scattering event, its probability increases with $Z 2^{2}$ as does the scattering cross section in eq. 3 . In contrast for heavy projectiles $E_{T h}$ depends mainly on the surface binding energy and $Y_{P h y s}$ in the high energy range varies strongly with the projectile to target mass ratio.

For the fusion energy range the sputter yield is dominated by the threshold energy in particular for $\mathrm{W}$ first wall components. For the case D-> W the typical energies are just around the threshold which is why $\mathrm{W}$ in a fusion experiment is typically not eroded by $\mathrm{D}$ but by the impact of heavier impurity ions like $\mathrm{O}$ or $\mathrm{C}$.

A physical model for the sputter yield is given by [5] which assumes that the sputter yield is given by the nuclear stopping cross section near the surface times a function describing the threshold behavior. In a simpler approach a general fit formula for the 


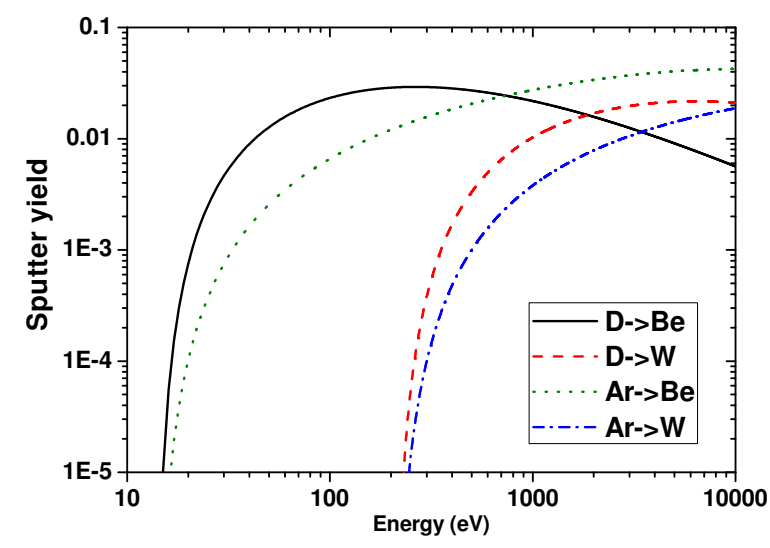

FIGURE 3. Projectile energy dependence of the physical sputter yield

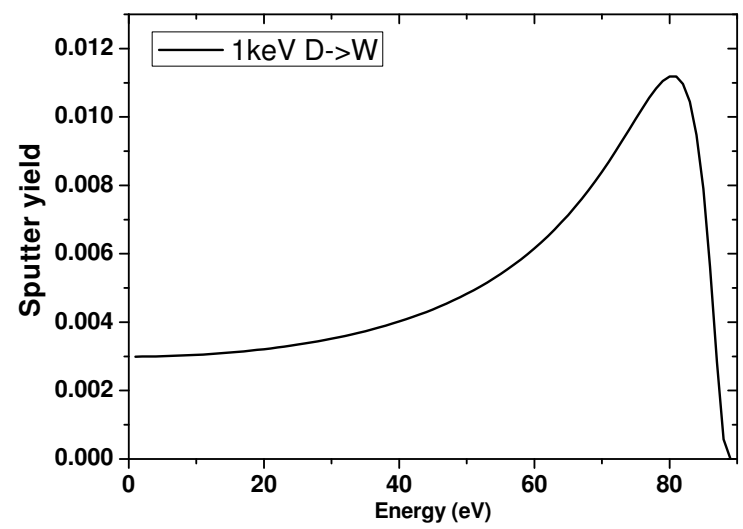

FIGURE 4. Example for dependence of $Y_{\text {Phys }}$ on the projectile angle of incidence with respect to the surface normal

energy dependence is given by Bohdansky [6]. It has two fit parameters: $E_{T h}$ and Q0 where $\mathrm{Q} 0$ is a basic scaling factor. These two fit parameters have been determined for a large number of projectile target combinations.

To sputter an atom from a surface an impulse pointing perpendicularly away from the surface has to be transferred from the projectile to the target. For perpendicular angle of incidence this requires at least two collision events where as for oblique angles of incidence enough impulse away from the surface can be transferred in a single collision. From this argument a dependence of $Y_{\text {Phys }}$ on the projectiles angle of incidence $\alpha$ relative to the surface normal can be expected: As $\alpha$ increases so does $Y_{\text {Phys }}$ until for very oblique angle reflection becomes dominant and $Y_{\text {Phys }}$ decreases to 0 for $\alpha=90$. A typical example for the angular dependence of the sputter yield is given in Fig. 4. $Y_{P h y s}$ initially 


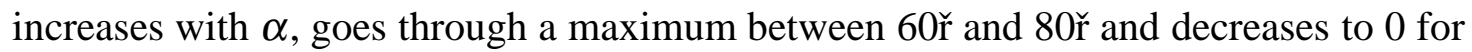
$\alpha=90$.

A model for the angular dependence is given by [7],[8]. It describes the total physical sputter yield as the product of the energy depedence times the angular dependence: $Y(E, \alpha)=Y(E) * Y(\alpha)$.

So far physical sputtering was described for a constant target composition that would not change during the bombardment. However this is generally not the case. For instance for projectiles that do not degas from the target after impact (as noble gases or hydrogen isotopes do) the projectile species concentration will build up in the surface diluting it and change the sputter yield. If the self sputtering yield of the depositing projectile is $<1$ eventually a layer will form shielding the underlying target surface from further erosion. The balance between net erosion and deposition in such a system can be quite narrow as is exemplified for the case of $\mathrm{W}$ sputtering by $\mathrm{C}$ and $\mathrm{D}$ in [9]

On the other hand if the target surface is made up from multiple elements which will usually have different sputter yields the relative concentrations of these components will vary under energetic particle bombardment. For a mixed material surface the so called partial sputter yield $Y_{i}^{\text {Part }}$ of an element can be calculated from the total sputter yield $Y_{i}^{T o t}$ of the element from a pure surface by scaling $Y_{i}^{T o t}$ with the concentration $C_{i}$

of the element in the surface: $Y_{i}^{\text {Part }} \approx C_{i} * Y_{i}^{\text {Tot }}$. This results in an equilibrium surface composition where the partial sputter yields of all components become equal.

\section{CHEMICAL EROSION}

The process of chemical erosion occurs when during exposure to a flux of reactive particles a target surface is eroded via the formation and effusion of volatile molecules. An example that is of particular interest is the chemical erosion of graphite by hydrogen isotopes. There the formation of volatile hydrocarbon species (dominantly $\mathrm{CH}_{4}$ ) results in erosion of the graphite surface. Due to the involved chemistry chemical erosion exhibits a strong temperature dependence. Further it does not require high energy projectiles but also occurs during exposure to a flux of thermal reactive atoms. This lack of a threshold energy is the major difference to physical sputtering.

For the erosion of graphite by atomic hydrogen a model exists that details the microscopic steps that lead to the formation of a volatile radical including the temperature dependence. This model is visualized in Fig. 5. Microscopic model

Kypers Cricle + Slide 23

Origin of temperature maximum

What is not included in the model

Different reaction products

Flux dependence

-> Still area of active research 


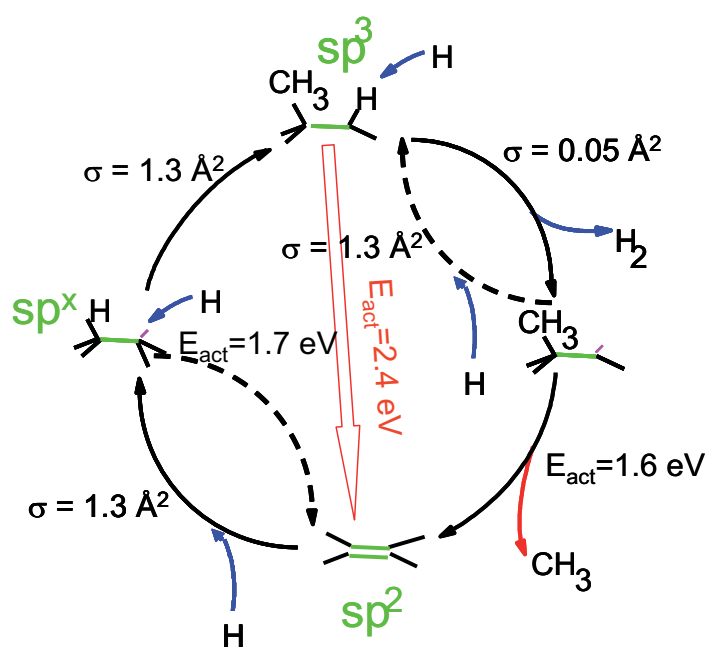

FIGURE 5. Schema of the microscopy model for chemical erosion "Küpers Kreisel"

\section{CHEMICAL SPUTTERING}

At first glance it is difficult to see the difference between chemical erosion and chemical sputtering. Chemical sputtering is a process whereby ion bombardment causes or allows a chemical reaction to occur which produces a particle that is weakly bound to the surface and hence easily desorbs in the gas phase. I.e for chemical sputtering energetic particles are needed to trigger a chemical reaction and therefore, in contrast to chemical erosion, it depends on the projectile energy. Often chemical erosion and chemical sputtering occur simultaneously as it is for instance the case during bombardment of graphite by hydrogen isotopes.

Some other prominent examples of chemical sputtering are: Bombardment of carbon by noble gas ions in the presence of atomic hydrogen; Bombardment of carbon by noble gas ions in the presence of molecular oxygen; Etching of silicon by fluorine plasmas which is by far the most important industrial plasma process.

For case of chemical sputtering of graphite by hydrogen isotopes a basic model exists and schematically shown in Fig. 6.

Synergistics between ion bombardment and reactive species

Example chemical sputtering by co-bombardment with Ar and H0

Effect of radiation damage + production of active sites

Complex temperature dependence + maximum with temperature

What is still not understood

Temperature dependence of maximum

decrease high fluence

-> Still area of active research 


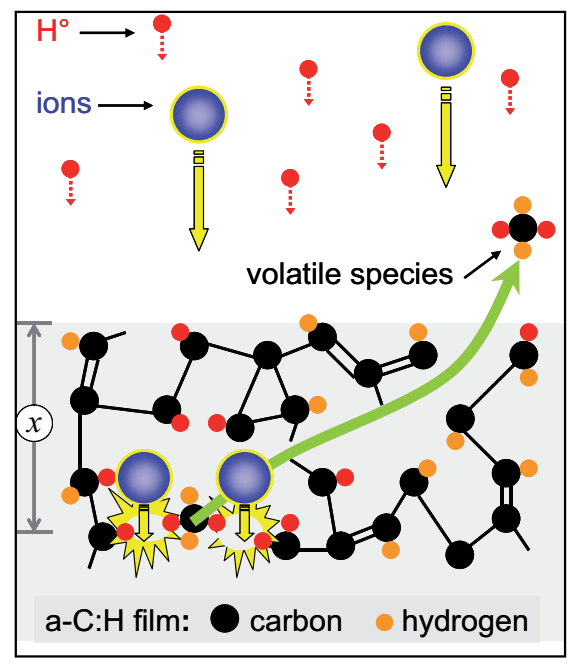

FIGURE 6. Visualization of the basic mechanism behind chemical sputtering of $\mathrm{C}$ by $\mathrm{H}$

\section{RADIATION ENHANCED SUBLIMATION}

Radiation enhanced sublimation (RES) is a process that was first established for the bombardment of highly oriented pyrolytic graphite (HOPG). Under bombardment by inert projectiles an erosion yield was found experimentally at temperatures $>1000 \mathrm{~K}$ that was significantly larger that the sum of the physical sputtering and normal sublimation. An investigation of the particle energy spectra during RES showed that the eroded particles had a thermal energy distribution [10]. This is in contrast to normal physically sputtered particles which feature an energy distribution from $\mathrm{eV}$ to the maximal transferable energy [11]. The underlying processes that lead to RES are described in [12] as the diffusion of weakly bonded interstitial carbon atoms to the surface where they sublime. The total RES yield depends on the ratio of two concurrent processes: Diffusion of interstitial atoms to the surface and recombination of the interstitials at vacancies. Under fusion conditions the high flux of incident particles generates a large abundance of vacancies which increases the loss of interstitials and thus reduces the RES yield. Further for low particle energies encountered for instance on the divertor region, very little or no radiation damage is produces and thus no interstitials are generated making the influence of RES on the total erosion yield negligible.

Recently [13] RES like enhancements of the erosion yield at high temperatures where also found for metals at the PISCES-B plasma simulator at UC-San Diego. In these experiments $\mathrm{Li}, \mathrm{Ga}, \mathrm{Be}$ and Au samples were exposed to a high flux $\left(\approx 10^{22}\left(\mathrm{~m}^{-2} \mathrm{~s}^{-1}\right)\right)$ low energy $(\approx 50 \mathrm{eV}) \mathrm{D}$ plasma. It was found that for both solid $(\mathrm{Be}, \mathrm{Au})$ and liquid $(\mathrm{Li}, \mathrm{Ga})$ metals the erosion rate increased exponentially for temperatures at which normal sublimation does not contribute significantly to the total erosion flux. In Fig. 7 the enhanced erosion of Be under high flux low energy D bombardment is shown as an example for RES in metals. It was further found in these experiments that at the onset of the enhanced erosion the average velocity of the atoms being removed from the sample decreases and that the enhanced erosion scales with the incident flux. This indicated that the enhanced 


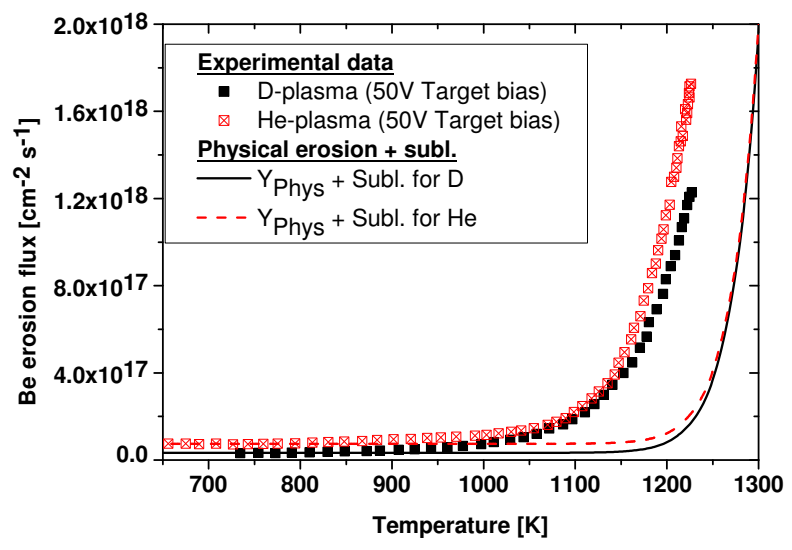

FIGURE 7. Example for RES in metals: High Temperature and flux erosion of Be with low projectile energy

erosion of metals is, similar to RES of C, due to sublimation of weakly bonded defect atoms generated during the bombardment. However due to the fast annealing of bulk defects in metals at elevated temperatures a different defect sublimation mechanism must be responsible for the enhanced erosion of metals. In [13] the sublimation of ad-atoms generated by the impact of the energetic particles from the plasma is suggested as a possible mechanism to explain the observed effect. An additional explanation is that at the high fluxes present in a plasma experiment the surface is supersaturated with $\mathrm{D}$ or $\mathrm{He}$ atoms which leads to a reduction of the surface binding energy and hence to an increase in sublimation [14]. Both models are based on the formation and sublimation of weakly bonded surface atoms due to defects generated by the high incident particle flux. So far the effect of thermally enhanced erosion of metals has only been confirmed in high flux plasma experiments. In an ion beam experiment which operates at particle fluxes that are at least 4 orders of magnitude lower that those encountered in a plasma experiment an enhancement in erosion is more difficult to observe because normal sublimation will dominate the total erosion flux from the sample already at elevated temperatures where the ad-atom sublimation flux in low. Also, the current model understanding requires a very high particle flux to create the weakly bonded surface defects in large enough quantities to counter the fast recombination of defects on metal surface, such that they can sublime at sufficient rates to give rise to enhanced erosion.

While RES of C does not significantly enhance the total erosion yield, RES of metals can dominate the erosion of metals under high flux low energy bombardment in a fusion experiment. 


\section{ACKNOWLEDGMENTS}

\section{REFERENCES}

1. M. Mayer, Technical report IPP 113 (1997).

2. J. Lindhard, and M. Scharff, Phys. Rev. 124, 128 (1961).

3. F. Bloch, Ann. Phys. (Leipzig) 16, 285 (1933).

4. P. C. Stangeby, The plasma boundary of magnetic fusion devices, IOP Pulishing, 2000.

5. P.Sigmund, Phys. Rev 184 No. 2, 184 (1969).

6. J. Bohdansky, Nucl. Inst. Meth. B 2, 587 (1984).

7. Y. Yamamura, Y. Itikawa, and N. Itoh, Nagoya, 1993.

8. W. Eckstein, and R. Preuss, J. Nucl. Mat 320, 209 (2003).

9. K. Schmid, and J. Roth, J. Nucl. Mat 313-316, 302 (2003).

10. E. Vietzke, K. Flaskamp, M. Hennes, and V. Philips, Nucl. Instrum. Methods B 2, 617 (1984).

11. W. Eckstein, Nucl. Instrum. Methods B 27, 78 (1987).

12. J. Roth, and W. Möller, Nucl. Instrum. Methods B 7/8, 788 (1985).

13. R. P. Doerner, S. I. Krasheninnikov, and K. Schmid, Nucl. Instrum. Methods B 7/8, 788 (1985).

14. K. Schmid, M. Baldwin, and R. Doerner, J. Nucl. Mat 348, 294 (2006). 